\title{
The Proposed Model of Suppliers Performance Measurement for Vendor Held Stock (VHS) in Mining Industry
}

\author{
Sri Wahyuni, Suparno, and Iwan Vanany \\ Department of Industrial and Systems Engineering, Institut Teknologi Sepuluh Nopember, Surabaya \\ E-mail: sriwahyuni.mustamal@yahoo.com
}

\begin{abstract}
Vendor Held Stock (VHS) is the storage and management a system for spare parts and other material must prepared and managed with the suppliers in case study. The case study in this research is in nickel mining company in Indonesia. The company's case study requires all suppliers should provide the spare parts and other materials in their warehouses. The purpose of this study is to develop VHS supplier's performance measurement system in case study. The key performance indicators (KPI's) in VHS's supplier performance measurement system are determined to ensure the buyer demand can be fulfilled in a timely, systematic and well organized with the correct quantity and maintained quality. The method used in measuring supplier performance is a combination of the Analytical Hierarchy Process (AHP), The Objective Matrix (OMAX) and Fishbone Diagram Methods. The results of this research are expected to obtain measurement models and find the root cause and solutions to the current problem to improve overall supplier performance.
\end{abstract}

Keywords-Analytical Hierarchy Process (AHP), Fishbone Diagram, Objective Matrix (OMAX), Vendor Held Stock (VHS).

\section{INTRODUCTION}

$\mathrm{P}$ T. $\mathrm{X}$ is a mining company that operates an open nickel mine in South Sulawesi. The main product produced is $78 \%$ Nickel Matte with a production of 75,000 metric tons/year (5\% of Nickel's supply in the world). Through investments in technology and logistics, this company guarantees efficiency, growth, and sustainability in its operations. The cessation of production activities caused by anything can cause a huge opportunity loss. In this case, good inventory management is very important to ensure the company's production activities run smoothly and as planned and can reduce opportunity loss. The importance of procurement in business activities is because generally $50 \%$ $70 \%$ of the sales value comes from raw materials and services purchased by the procurement and purchasing department. So that the raw materials and services purchased, a large effect on corporate business profits (Weele A.J., 2010). Various attempts were made by the company to produce good inventory management. In the procurement of goods, the company implements several systems including the Vendor Held Stock (VHS) Agreement. This system serves to regulate the demand or stock of goods between the company and suppliers whose expectations can be done on time, systematically, arranged with the correct quantity and quality that is maintained by requiring suppliers to provide several goods in the supplier's warehouse. The application of this

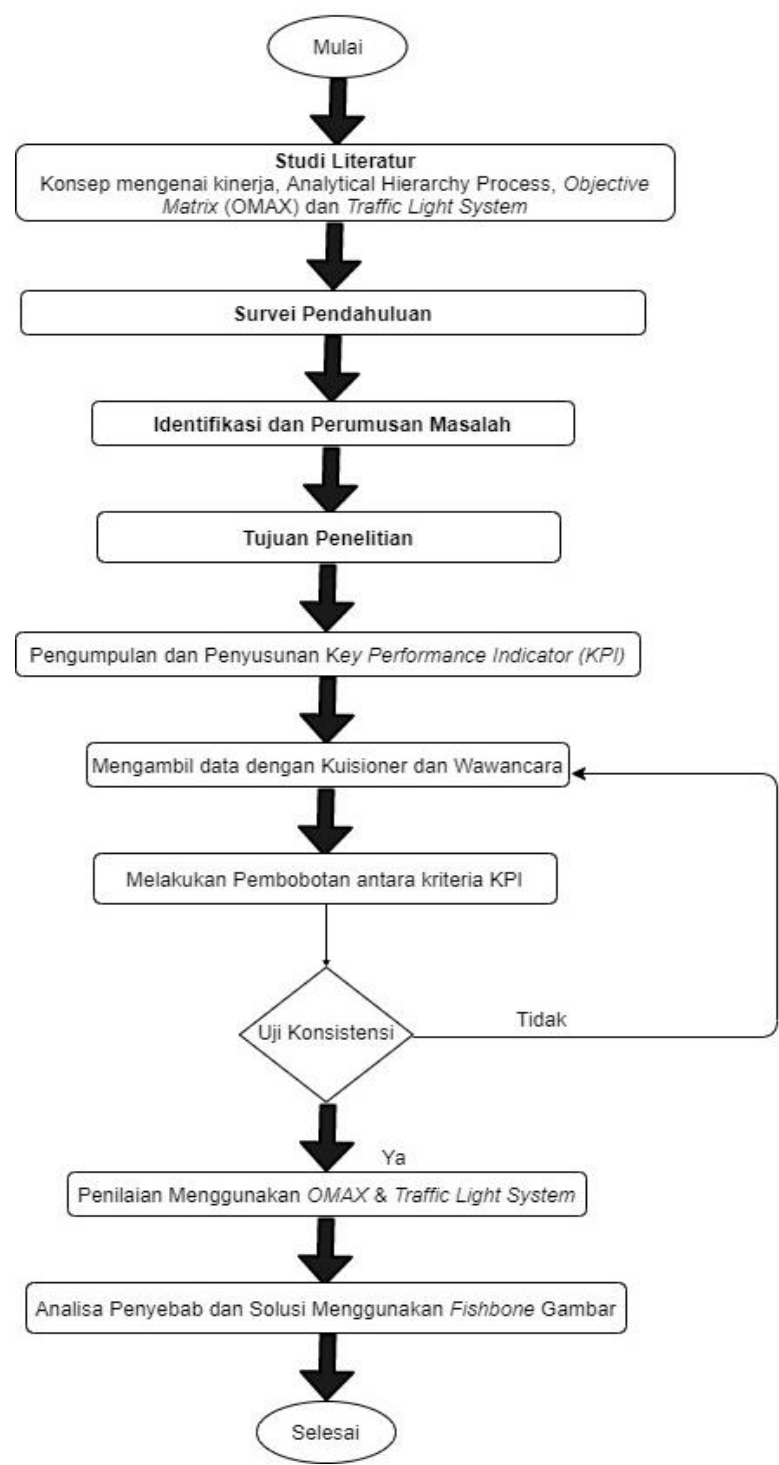

Figure 1. Research step.

system has involved many local suppliers and made them professional in their activities as work partners. In increasing its productivity, performance measurements from suppliers have been carried out every year. However, the results of these measurements have not been able to improve overall performance. Seeing these conditions, it is necessary to change the measurement model that can show the real performance of the Vendor Held Stock (VHS) supplier so that it can be evaluated and improved in the future. This research will use the criteria that have been used by the company as 
The $6^{\text {th }}$ International Seminar on Science and Technology (ISST) 2020

July $25^{\text {th }} 2020$, Institut Teknologi Sepuluh Nopember, Surabaya, Indonesia

Table 1.

Formatting rules

\begin{tabular}{lll}
\hline \hline Criteria & Element & KPI \\
\hline On Time Services & Fast Delivery & LT \\
& Stock Available & SOH \\
Safety & PPE (Personnel Protection Equipment) comply & PPE \\
& Follow the Safety Regulation & SR \\
Quality & Complain from user & QM \\
& Genuine Part & OM \\
After Sales Service & Standard Packaging & PM \\
& Visit user or related department & VS \\
Response Time & Training / Workshop & TS \\
& How Fast to Respond All Question & TQ \\
Reporting and Admin & How Fast to Solve The Problem & TP \\
& Outstanding Reservation Report & RR \\
Compliance & Stock OnHand Report & SR \\
& Office Consistent in tidy condition & AO \\
\hline \hline
\end{tabular}

Table 2.

Results of determination of criteria and sub-criteria

\begin{tabular}{|c|c|c|c|c|c|c|c|c|}
\hline \multirow[b]{2}{*}{ Criteria } & \multirow[b]{2}{*}{ No. } & \multirow{2}{*}{ Sub-Kriteria } & \multicolumn{3}{|c|}{ Responden } & \multicolumn{2}{|c|}{ Pilihan Jawaban } & \multirow[b]{2}{*}{ Total } \\
\hline & & & 1 & 2 & 3 & Penting & Tidak Penting & \\
\hline \multirow{2}{*}{ On Time Services } & 1 & On Time Delivery & $\mathrm{P}$ & $\mathrm{P}$ & $\mathrm{P}$ & 3 & 0 & 3 \\
\hline & 2 & Stock Available & $\mathrm{P}$ & $\mathrm{P}$ & $\mathrm{P}$ & 3 & 0 & 3 \\
\hline \multirow{3}{*}{ Safety } & 3 & PPE (Personal Protection Equipment) Comply & $\mathrm{P}$ & $\mathrm{P}$ & $\mathrm{P}$ & 3 & 0 & 3 \\
\hline & 4 & Follow the Safety Regulation & $\mathrm{P}$ & $\mathrm{P}$ & $\mathrm{P}$ & 3 & 0 & 3 \\
\hline & 5 & Complain from User & $\mathrm{P}$ & $\mathrm{P}$ & $\mathrm{P}$ & 3 & 0 & 3 \\
\hline \multirow{2}{*}{ Quality } & 6 & Genuine Part & $\mathrm{P}$ & $\mathrm{P}$ & $\mathrm{P}$ & 3 & 0 & 3 \\
\hline & 7 & Standard Packaging & $\mathrm{P}$ & $\mathrm{P}$ & $\mathrm{P}$ & 3 & 0 & 3 \\
\hline \multirow{2}{*}{ After Sales Service } & 8 & Visit to User Procurement or Related Department & TP & $\mathrm{P}$ & $\mathrm{P}$ & 2 & 1 & 3 \\
\hline & 9 & Training/Workshop & TP & $\mathrm{P}$ & $\mathrm{TP}$ & 0 & 3 & 3 \\
\hline \multirow{3}{*}{ Response Time } & 10 & How Fast to Respond All Enquines & $\mathrm{P}$ & $\mathrm{TP}$ & $\mathrm{TP}$ & 1 & 2 & 3 \\
\hline & 11 & How Fast to Resolve the Problem & $\mathrm{P}$ & $\mathrm{P}$ & - & 2 & 0 & 2 \\
\hline & 12 & Outstanding Reservation Report & $\mathrm{P}$ & $\mathrm{P}$ & $\mathrm{P}$ & 3 & 0 & 3 \\
\hline \multirow[t]{2}{*}{ Reporting \& Admin } & 13 & Stock on Hand Report & $\mathrm{P}$ & $\mathrm{P}$ & $\mathrm{P}$ & 3 & 0 & 3 \\
\hline & 14 & Office Consistant in Tidy Condition & $\mathrm{P}$ & $\mathrm{P}$ & $\mathrm{P}$ & 3 & 0 & 3 \\
\hline \multirow{2}{*}{ Compliance } & 15 & Follow All Applicable Government & $\mathrm{P}$ & $\mathrm{P}$ & $\mathrm{P}$ & 3 & 0 & 3 \\
\hline & 16 & Violations of Government Rules & $\mathrm{P}$ & $\mathrm{P}$ & $\mathrm{P}$ & 3 & 0 & 3 \\
\hline $\begin{array}{l}\text { Other Criteria } \\
\text { Resources }\end{array}$ & 17 & Improve Resources from Local Community & $\mathrm{P}$ & $\mathrm{P}$ & $\mathrm{P}$ & 3 & 0 & 3 \\
\hline
\end{tabular}

the main source and process the weight calculation by combining the methods of achieving performance and analyzing problems that might occur. The productivity measurement method used is the Analytical Hierarchy Process (AHP) method combined with the Objective Matrix (OMAX) and Fishbone Diagram method.

\section{ANALYTICAL HIERARCHY PROCESS (AHP) DAN OBJECTIVE MATRIX (OMAX)}

\section{A. Analytical Hierarchy Process (AHP)}

Analytical Hierarchy Process (AHP) according to Saaty in Syaifullah (2010), is a decision support model that will break down complex multi-criteria problems into a hierarchy. Hierarchy is defined as a representation of a complex problem in a multilevel structure where the first level is the goal, then followed by the level of criteria, sub-criteria, and so on to the last level, namely alternatives. In the application of supplier, selection requires a systematic process of identification, determining priority criteria, and measuring relevant performance criteria (Tam and Tummala, 2000). The use of the Analytical Hierarchy Process (AHP) methods as an approach for selecting and evaluating supplier performance (Narasimhan, 1983). This method can accommodate the assessment criteria that are qualitative and quantitative and easily understood and applied.

\section{B. Objective Matrix (OMAX)}

Objective Matrix (OMAX) is a partial productivity measurement system developed to monitor productivity in every part of the company with productivity criteria that are consistent with the existence of that part (objective). This method was developed by Dr. James L. Riggs (Department of Industrial Engineering at Oregon State University).

The uses of OMAX are:

a. As a means of measuring productivity and performance

b. As a tool for solving productivity and performance problems

c. Monitoring tools for productivity and performance needs By using a performance measurement system based on the AHP and OMAX methods, companies can monitor all aspects of supplier performance and immediately take corrective and preventive actions to fix the performance of suppliers who are still at a low level.

\section{Fishbone Diagram}

Fishbone Diagrams are also known as Cause-Effect Diagrams. This tool was invented by a Japanese quality control expert, Dr. Ishikawa Kaoru. Fishbone diagrams can 
The $6^{\text {th }}$ International Seminar on Science and Technology (ISST) 2020

July $25^{\text {th }} 2020$, Institut Teknologi Sepuluh Nopember, Surabaya, Indonesia

Table 3.

Results of weighting of criteria

\begin{tabular}{lll}
\hline \hline Criteria & Weight & Consistency Ratio \\
\hline On Time Services & 0.137 & \\
Safety & 0.201 & \\
Quality & 0.206 & $1 \%$ \\
After Sales Service & 0.089 & \\
Response Time & 0.102 & \\
Reporting and Admin & 0.058 & \\
Compliance & 0.128 & \\
Resources & 0.080 & \\
\hline \hline
\end{tabular}

Table 4.

Weighting results for criteria and sub-criteria

\begin{tabular}{|c|c|c|c|c|c|c|c|c|c|c|c|c|c|c|c|}
\hline Criteria & $\begin{array}{l}\text { On } \\
\text { Service }\end{array}$ & $e^{\text {Time }}$ & \multicolumn{2}{|l|}{ Safety } & \multicolumn{3}{|c|}{ Quality } & \multirow{2}{*}{$\begin{array}{l}\text { After } \\
\text { Sales } \\
\text { Services } \\
0.089\end{array}$} & \multirow{2}{*}{$\begin{array}{l}\begin{array}{l}\text { Response } \\
\text { Time }\end{array} \\
0.102\end{array}$} & \multicolumn{3}{|c|}{ Report \& Admin } & \multicolumn{2}{|c|}{ Compliance } & Resource \\
\hline $\begin{array}{l}\text { Weight } \\
\text { Criteria }\end{array}$ & 0.137 & & 0.201 & & 0.206 & & & & & 0.058 & & & 0.128 & & 0.080 \\
\hline Sub Criteria & LT & $\mathrm{SOH}$ & PPE & SR & QM & $\mathrm{OM}$ & PM & & & $\mathrm{RR}$ & SR & $\mathrm{AO}$ & GR & VR & \\
\hline $\begin{array}{l}\text { Weight } \\
\text { Normatif }\end{array}$ & 0.78 & 0.22 & 0.12 & 0.88 & 0.11 & 0.83 & 0.06 & & & 0.64 & 0.23 & 0.13 & 0.97 & 0.03 & \\
\hline $\begin{array}{l}\text { Total Weight } \\
\text { Weight } \\
\text { Relative }\end{array}$ & $\begin{array}{l}1.00 \\
0.11\end{array}$ & 0.03 & $\begin{array}{l}1.00 \\
0.02\end{array}$ & 0.18 & $\begin{array}{l}1.00 \\
0.02\end{array}$ & 0.17 & 0.01 & & & $\begin{array}{l}1.00 \\
0.04\end{array}$ & 0.01 & 0.01 & $\begin{array}{l}1.00 \\
0.12\end{array}$ & 0.00 & \\
\hline Total Weight & 0.137 & & 0.201 & & 0.206 & & & 0.089 & 0.102 & 0.058 & & & 0.128 & & 0.080 \\
\hline
\end{tabular}

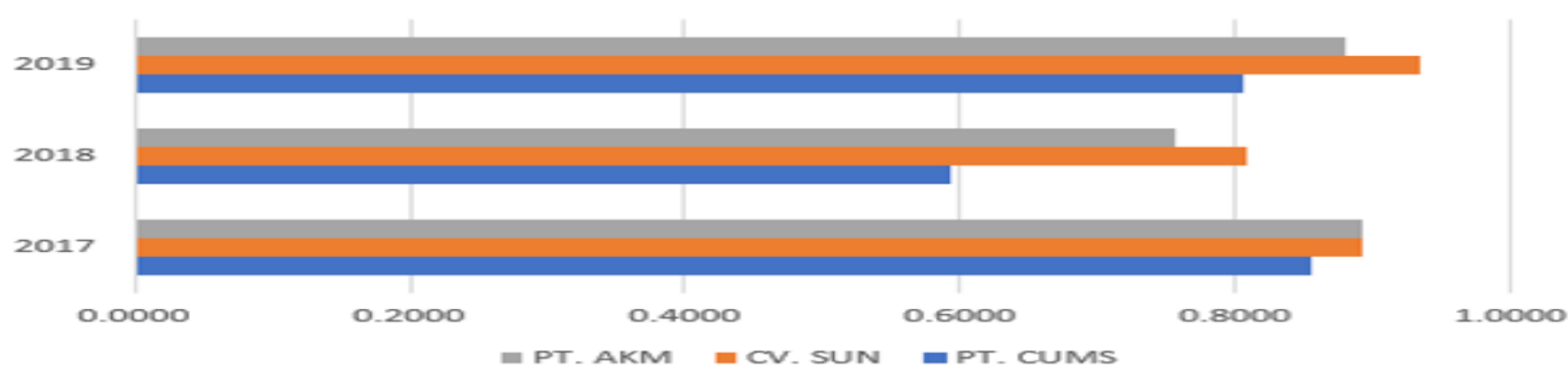

Figure 2. Supplier performance 2017-2019.

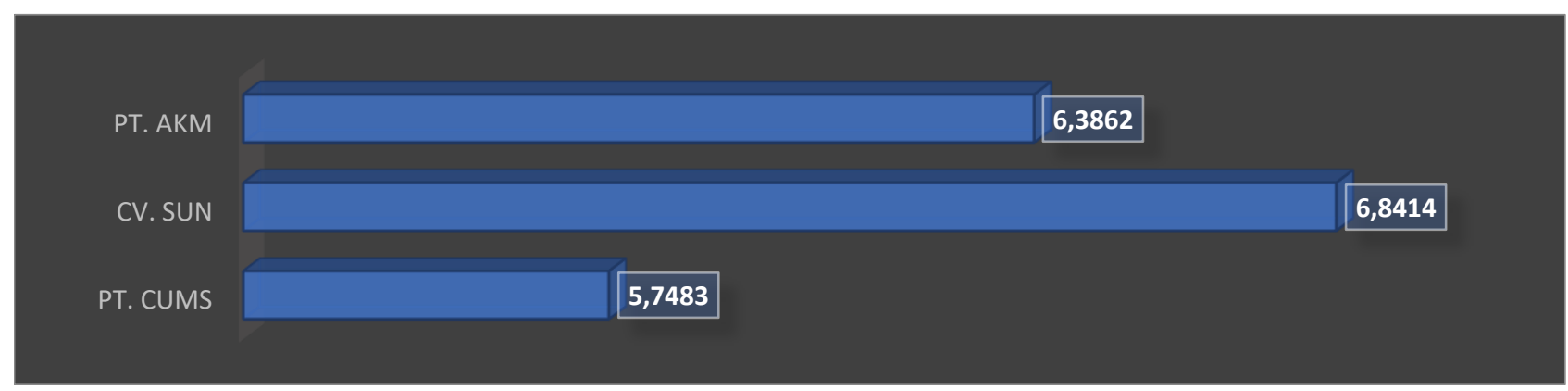

Figure 3. Recapitulation calculate of OMAX.

help identify possible causes of problems and sort ideas into more specific categories. This tool makes a difference between cause and effect.

Fishbone Diagrams allow companies to look at all the causal factors and find the main root of the problem. The steps in conducting a Fishbone analysis are agreeing to the problem statement, identifying the categories that exist, finding potential causes, examining and agreeing on the most probable causes.

\section{METHOD}

The data used in this study is testing the importance of the criteria and sub-criteria owned by PT.X by using quantitative methods in the form of questionnaires and interviews. Also, data were obtained from the results of the performance of three VHS suppliers for the period January 2017 - December 2019 relating to the provision of Welding \& Safety Consumables (PT. CUMS, CV. SUN, and PT. AKM).

Weights data used for supplier performance evaluation are obtained from filling out the questionnaire using the AHP method. After weighting each criterion can be obtained, the data is processed using OMAX and the traffic light system. From the problems found in OMAX images, then analyzed using Fishbone diagrams. The stages carried out in this study are as a Figure 1. The criteria used by PT. $\mathrm{X}$ in evaluating supplier performance is in Table 1. 
The $6^{\text {th }}$ International Seminar on Science and Technology (ISST) 2020

Table 5.

Result supplier performance period 2017-2019

\begin{tabular}{lllllll}
\hline \hline \multirow{2}{*}{ No } & \multirow{2}{*}{ Vendor } & \multicolumn{3}{c}{ KPI (Years) } & \multirow{2}{*}{ Average } & Min \\
\cline { 3 - 7 } & & 2017 & 2018 & 2019 & 0.7514 & 0.5935 \\
2 & PT. CUMS & 0.8552 & 0.5935 & 0.8054 & 0.8748 & 0.8072 \\
3 & CV. SUN & 0.8915 & 0.8072 & 0.9348 & 0.8778 & 0.7566 \\
\hline \hline
\end{tabular}

Table 6.

Data key performance indicator PT. CUMS

\begin{tabular}{|c|c|c|c|c|c|c|}
\hline \multirow[b]{2}{*}{ Productivity Criteria } & \multirow[b]{2}{*}{ KPI No } & \multirow[b]{2}{*}{ KPI } & \multicolumn{3}{|c|}{1 Jan 2017 - 31 Dec 2019} & \multirow{2}{*}{\begin{tabular}{|c|} 
Measured \\
performance \\
on 31 Dec 2019
\end{tabular}} \\
\hline & & & \begin{tabular}{|c|} 
The worst \\
performance
\end{tabular} & \begin{tabular}{|c|} 
Expected \\
performance
\end{tabular} & \begin{tabular}{c|} 
Based \\
performance on 2017
\end{tabular} & \\
\hline \multirow[t]{2}{*}{ Ketepatan Pelayanan } & KPI 1 & Kecepatan Pengiriman & 0.1021 & 0.1500 & 0.1216 & 0.1021 \\
\hline & KPI 2 & Ketersediaan Barang & 0.1021 & 0.1500 & 0.1216 & 0.1021 \\
\hline \multirow[t]{2}{*}{ Keselamatan } & KPI 3 & Penggunaan APD (Alat Pelindung Diri) & 0.0000 & 0.0500 & 0.0500 & 0.0500 \\
\hline & KPI 4 & Mematuhi Peraturan Keselamatan & 0.0000 & 0.0500 & 0.0500 & 0.0500 \\
\hline \multirow[t]{3}{*}{ Kualitas } & KPI 5 & Keluhan Kualitas Dari Pengguna & 0.0450 & 0.1200 & 0.1150 & 0.1150 \\
\hline & KPI 6 & Keaslian Barang & 0.0000 & 0.0600 & 0.0600 & 0.0600 \\
\hline & KPI 7 & Kemasan Barang & 0.0190 & 0.0200 & 0.0190 & 0.0190 \\
\hline Pelayanan Purna Jual & KPI 8 & Frekuensi Kunjungan ke Pengguna & 0.0420 & 0.1000 & 0.0420 & 0.0420 \\
\hline Waktu Tanggapan & KPI 9 & Kecepatan Mengatasi Masalah & 0.0800 & 0.1000 & 0.0900 & 0.0900 \\
\hline \multirow[t]{3}{*}{ Laporan dan Administrasi } & KPI 10 & Frekuensi Pengiriman Laporan Reservasi & 0.0207 & 0.0350 & 0.0280 & 0.0207 \\
\hline & KPI 11 & Frekuensi Pengiriman Laporan SOH & 0.0245 & 0.0350 & 0.0280 & 0.0245 \\
\hline & KPI 12 & Konsisten Kerapihan Administrasi Kantor & 0.0300 & 0.0300 & 0.0300 & 0.0300 \\
\hline \multirow[t]{2}{*}{ Tingkat Pemenuhan } & KPI 13 & Mengikuti Aturan Pemerintah & 0.0500 & 0.0500 & 0.0500 & 0.0500 \\
\hline & KPI 14 & Pelanggaran Terhadap Aturan Pemerintah & 0.0500 & 0.0500 & 0.0500 & 0.0500 \\
\hline Sumber Daya & KPI 15 & $\begin{array}{l}\text { Tingkat Penggunaan Sumber Daya dari } \\
\text { Komunitas Lokal }\end{array}$ & NA & NA & NA & NA \\
\hline
\end{tabular}

Table 7.

Scoring OMAX and traffic light system PT. CUMS

\begin{tabular}{|c|c|c|c|c|c|c|c|c|c|c|c|c|c|c|c|c|}
\hline \multicolumn{2}{|c|}{ Performance Kriteria } & KPI 1 & KPI 2 & KPI 3 & KPI 4 & KPI 5 & KPI 6 & KPI 7 & KPI 8 & KPI 9 & KPI 10 & KPI 11 & KPI 12 & KPI 13 & KPI 14 & KPI 15 \\
\hline \multicolumn{2}{|c|}{ Performance } & 0.1021 & 0.1021 & 0.0500 & 0.0500 & 0.1150 & 0.0600 & 0.0190 & 0.0420 & 0.0900 & 0.0207 & 0.0245 & 0.0300 & 0.0500 & 0.0500 & 0.0000 \\
\hline & 10 & 0.1500 & 0.1500 & 0.0500 & 0.0500 & 0.1200 & 0.0600 & 0.0200 & 0.1000 & 0.1000 & 0.0350 & 0.0350 & 0.0300 & 0.0500 & 0.0500 & 0.0000 \\
\hline & 9 & 0.1459 & 0.1459 & 0.0500 & 0.0500 & 0.1193 & 0.0600 & 0.0199 & 0.0917 & 0.0986 & 0.0340 & 0.0340 & 0.0300 & 0.0500 & 0.0500 & 0.0000 \\
\hline & 8 & 0.1419 & 0.1419 & 0.0500 & 0.0500 & 0.1186 & 0.0600 & 0.0197 & 0.0834 & 0.0971 & 0.0330 & 0.0330 & 0.0300 & 0.0500 & 0.0500 & 0.0000 \\
\hline $\mathrm{s}$ & 7 & 0.1378 & 0.1378 & 0.0500 & 0.0500 & 0.1179 & 0.0600 & 0.0196 & 0.0751 & 0.0957 & 0.0320 & 0.0320 & 0.0300 & 0.0500 & 0.0500 & 0.0000 \\
\hline $\mathrm{C}$ & 6 & 0.1338 & 0.1338 & 0.0500 & 0.0500 & 0.1171 & 0.0600 & 0.0194 & 0.0669 & 0.0943 & 0.0310 & 0.0310 & 0.0300 & 0.0500 & 0.0500 & 0.0000 \\
\hline $\mathrm{O}$ & 5 & 0.1297 & 0.1297 & 0.0500 & 0.0500 & 0.1164 & 0.0600 & 0.0193 & 0.0586 & 0.0929 & 0.0300 & 0.0300 & 0.0300 & 0.0500 & 0.0500 & 0.0000 \\
\hline $\mathrm{R}$ & 4 & 0.1257 & 0.1257 & 0.0500 & 0.0500 & 0.1157 & 0.0600 & 0.0191 & 0.0503 & 0.0914 & 0.0290 & 0.0290 & 0.0300 & 0.0500 & 0.0500 & 0.0000 \\
\hline \multirow[t]{4}{*}{ E } & 3 & 0.1216 & 0.1216 & 0.0500 & 0.0500 & 0.1150 & 0.0600 & 0.0190 & 0.0420 & 0.0900 & 0.0280 & 0.0280 & 0.0300 & 0.0500 & 0.0500 & 0.0000 \\
\hline & 2 & 0.1151 & 0.1151 & 0.0333 & 0.0333 & 0.0917 & 0.0400 & 0.0190 & 0.0420 & 0.0867 & 0.0256 & 0.0268 & 0.0300 & 0.0500 & 0.0500 & 0.0000 \\
\hline & 1 & 0.1086 & 0.1086 & 0.0167 & 0.0167 & 0.0683 & 0.0200 & 0.0190 & 0.0420 & 0.0833 & 0.0231 & 0.0257 & 0.0300 & 0.0500 & 0.0500 & 0.0000 \\
\hline & 0 & 0.1021 & 0.1021 & 0.0000 & 0.0000 & 0.0450 & 0.0000 & 0.0190 & 0.0420 & 0.0800 & 0.0207 & 0.0245 & 0.0300 & 0.0500 & 0.0500 & 0.0000 \\
\hline \multicolumn{2}{|l|}{ Score } & 0 & 0 & 10 & 10 & 3 & 10 & 3 & 3 & 3 & 0 & 0 & 10 & 10 & 10 & 0 \\
\hline \multicolumn{2}{|c|}{ Weight } & 0.106 & 0.031 & 0.024 & 0.177 & 0.023 & 0.171 & 0.012 & 0.089 & 0.102 & 0.037 & 0.013 & 0.007 & 0.124 & 0.004 & 0.080 \\
\hline \multicolumn{2}{|l|}{ Value } & 0.0000 & 0.0000 & 0.2368 & 1.7720 & 0.0692 & 1.7083 & 0.0362 & 0.2681 & 0.3054 & 0.0000 & 0.0000 & 0.0736 & 1.2373 & 0.0412 & 0.0000 \\
\hline \multicolumn{2}{|c|}{ Total Value } & \multicolumn{15}{|c|}{483} \\
\hline
\end{tabular}

Table 8.

Data key performance indicator CV. SUN

\begin{tabular}{|c|c|c|c|c|c|c|}
\hline \multirow[b]{2}{*}{ Productivity Criteria } & \multirow[b]{2}{*}{ KPI No } & \multirow[b]{2}{*}{ KPI } & \multicolumn{3}{|c|}{1 Jan 2017 - 31 Dec 2019} & \multirow{2}{*}{\begin{tabular}{|c|} 
Measured \\
performance \\
on 31 Dec 2019 \\
\end{tabular}} \\
\hline & & & \begin{tabular}{|c|} 
The worst \\
performance
\end{tabular} & $\begin{array}{c}\text { Expected } \\
\text { performance }\end{array}$ & $\begin{array}{c}\text { Based } \\
\text { performance on } 2017\end{array}$ & \\
\hline \multirow[t]{2}{*}{ Ketepatan Pelayanan } & KPI 1 & Kecepatan Pengiriman & 0.1095 & 0.1500 & 0.1095 & 0.1305 \\
\hline & KPI 2 & Ketersediaan Barang & 0.1095 & 0.1500 & 0.1095 & 0.1305 \\
\hline \multirow[t]{2}{*}{ Keselamatan } & KPI 3 & Penggunaan APD (Alat Pelindung Diri) & 0.0000 & 0.0500 & 0.0500 & 0.0500 \\
\hline & KPI 4 & Mematuhi Peraturan Keselamatan & 0.0000 & 0.0500 & 0.0500 & 0.0500 \\
\hline \multirow[t]{3}{*}{ Kualitas } & KPI 5 & Keluhan Kualitas Dari Pengguna & 0.1180 & 0.1200 & 0.1190 & 0.1193 \\
\hline & KPI 6 & Keaslian Barang & 0.0600 & 0.0600 & 0.0600 & 0.0600 \\
\hline & KPI 7 & Kemasan Barang & 0.0190 & 0.0200 & 0.0190 & 0.0193 \\
\hline Pelayanan Purna Jual & KPI 8 & Frekuensi Kunjungan ke Pengguna & 0.0720 & 0.1000 & 0.0800 & 0.0800 \\
\hline Waktu Tanggapan & KPI 9 & Kecepatan Mengatasi Masalah & 0.0970 & 0.1000 & 0.0980 & 0.0980 \\
\hline \multirow{3}{*}{ Laporan dan Administrasi } & KPI 10 & Frekuensi Pengiriman Laporan Reservasi & 0.0280 & 0.0350 & 0.0333 & 0.0336 \\
\hline & KPI 11 & Frekuensi Pengiriman Laporan SOH & 0.0280 & 0.0350 & 0.0333 & 0.0336 \\
\hline & KPI 12 & Konsisten Kerapihan Administrasi Kantor & 0.0300 & 0.0300 & 0.0300 & 0.0300 \\
\hline \multirow[t]{2}{*}{ Tingkat Pemenuhan } & KPI 13 & Mengikuti Aturan Pemerintah & 0.0500 & 0.0500 & 0.0500 & 0.0500 \\
\hline & KPI 14 & Pelanggaran Terhadap Aturan Pemerintah & 0.0500 & 0.0500 & 0.0500 & 0.0500 \\
\hline Sumber Daya & KPI 15 & $\begin{array}{l}\text { Tingkat Penggunaan Sumber Daya dari } \\
\text { Komunitas Lokal }\end{array}$ & NA & NA & NA & NA \\
\hline
\end{tabular}


The $6^{\text {th }}$ International Seminar on Science and Technology (ISST) 2020

July $25^{\text {th }} 2020$, Institut Teknologi Sepuluh Nopember, Surabaya, Indonesia

Table 9 .

Scoring OMAX and traffic light system CV. SUN

\begin{tabular}{|c|c|c|c|c|c|c|c|c|c|c|c|c|c|c|c|c|}
\hline \multicolumn{2}{|c|}{ Performance Kriteria } & & & & & & & & & & & & & & & $\mathrm{PI}$ \\
\hline \multicolumn{2}{|c|}{ Performance } & 0.1305 & 0.1305 & 0.0500 & 0.0500 & 0.1193 & 0.0600 & 0.0193 & 0.0800 & 0.0980 & 0.0336 & 0.0336 & 0.0300 & 0.0500 & 0.0500 & 0.0000 \\
\hline & 10 & 0.1500 & 0.1500 & 0.0500 & 0.0500 & 0.1200 & 0.0600 & 0.0200 & 0.1000 & 0.1000 & 0.0350 & 0.0350 & 0.0300 & 0.0500 & 0.0500 & 0.0000 \\
\hline & 9 & 0.1442 & 0.1442 & 0.0500 & 0.0500 & 0.1199 & 0.0600 & 0.0199 & 0.0971 & 0.0997 & 0.0348 & 0.0348 & 0.0300 & 0.0500 & 0.0500 & .0000 \\
\hline & 8 & 0.1384 & 0.1384 & 0.0500 & 0.0500 & 0.1197 & 0.0600 & 0.0197 & 0.0943 & 0.0994 & 0.0345 & 0.0345 & 0.0300 & 0.0500 & 0.0500 & .0000 \\
\hline $\mathrm{S}$ & 7 & 0.1326 & 0.1326 & 0.0500 & 0.0500 & 0.1196 & 0.0600 & 0.0196 & 0.0914 & 0.0991 & 0.0343 & 0.0343 & 0.0300 & 0.0500 & 0.0500 & 0.0000 \\
\hline $\mathrm{C}$ & 6 & 0.1269 & 0.1269 & 0.0500 & 0.0500 & 0.1194 & 0.0600 & 0.0194 & 0.0886 & 0.0989 & 0.0340 & 0.0340 & 0.0300 & 0.0500 & 0.0500 & 0.0000 \\
\hline $\mathrm{O}$ & 5 & 0.1211 & 0.1211 & 0.0500 & 0.0500 & 0.1193 & 0.0600 & 0.0193 & 0.0857 & 0.0986 & 0.0338 & 0.0338 & 0.0300 & 0.0500 & 0.0500 & 0.0000 \\
\hline $\mathrm{R}$ & 4 & 0.1153 & 0.1153 & 0.0500 & 0.0500 & 0.1191 & 0.0600 & 0.0191 & 0.0829 & 0.0983 & 0.0335 & 0.0335 & 0.0300 & 0.0500 & 0.0500 & 0.0000 \\
\hline \multirow[t]{4}{*}{$\mathrm{E}$} & 3 & 0.1095 & 0.1095 & 0.0500 & 0.0500 & 0.1190 & 0.0600 & 0.0190 & 0.0800 & 0.0980 & 0.0333 & 0.0333 & 0.0300 & 0.0500 & 0.0500 & 0.0000 \\
\hline & 2 & 0.1095 & 0.1095 & 0.0333 & 0.0333 & 0.1187 & 0.0600 & 0.0190 & 0.0773 & 0.0977 & 0.0315 & 0.0315 & 0.0300 & 0.0500 & 0.0500 & 0.0000 \\
\hline & 1 & 0.1095 & 0.1095 & 0.0167 & 0.0167 & 0.1183 & 0.0600 & 0.0190 & 0.0747 & 0.0973 & 0.0298 & 0.0298 & 0.0300 & 0.0500 & 0.0500 & 0.0000 \\
\hline & 0 & 0.1095 & 0.1095 & 0.0000 & 0.0000 & 0.1180 & 0.0600 & 0.0190 & 0.0720 & 0.0970 & 0.0280 & 0.0280 & 0.0300 & 0.0500 & 0.0500 & 0.0000 \\
\hline \multicolumn{2}{|l|}{ Score } & 6 & 6 & 10 & 10 & 5 & 10 & 5 & 3 & 3 & 4 & 4 & 10 & 10 & 10 & 0 \\
\hline \multicolumn{2}{|c|}{ Weight } & 0.106 & 0.031 & 0.024 & 0.177 & 0.023 & 0.171 & 0.012 & 0.089 & 0.102 & 0.037 & 0.013 & 0.007 & 0.124 & 0.004 & 0.080 \\
\hline \multicolumn{2}{|l|}{ Value } & 0.6380 & 0.1842 & 0.2368 & 1.7720 & 0.1154 & 1.7083 & 0.0604 & 0.2681 & 0.3054 & 0.1468 & 0.0538 & 0.0736 & 1.2373 & 0.0412 & 0.0000 \\
\hline \multicolumn{2}{|c|}{ Total } & \\
\hline
\end{tabular}

Table 10.

Data key performance indicator PT. AKM

\begin{tabular}{|c|c|c|c|c|c|c|}
\hline \multirow[b]{2}{*}{ Productivity Criteria } & \multirow[b]{2}{*}{ KPI No } & \multirow[b]{2}{*}{ KPI } & \multicolumn{3}{|c|}{1 Jan 2017 - 31 Dec 2019} & \multirow{2}{*}{\begin{tabular}{|c|} 
Measured \\
performance \\
on 31 Dec 2019
\end{tabular}} \\
\hline & & & \begin{tabular}{|c|} 
The worst \\
performance
\end{tabular} & \begin{tabular}{|c|} 
Expected \\
performance
\end{tabular} & \begin{tabular}{|c|} 
Based \\
performance on 2017
\end{tabular} & \\
\hline \multirow[t]{2}{*}{ Ketepatan Pelayanan } & KPI 1 & Kecepatan Pengiriman & 0.1020 & 0.1500 & 0.1140 & 0.1080 \\
\hline & KPI 2 & Ketersediaan Barang & 0.1020 & 0.1500 & 0.1140 & 0.1080 \\
\hline \multirow[t]{2}{*}{ Keselamatan } & KPI 3 & Penggunaan APD (Alat Pelindung Diri) & 0.0000 & 0.0500 & 0.0500 & 0.0500 \\
\hline & KPI 4 & Mematuhi Peraturan Keselamatan & 0.0000 & 0.0500 & 0.0500 & 0.0500 \\
\hline \multirow[t]{3}{*}{ Kualitas } & KPI 5 & Keluhan Kualitas Dari Pengguna & 0.1190 & 0.1200 & 0.1190 & 0.1195 \\
\hline & KPI 6 & Keaslian Barang & 0.0600 & 0.0600 & 0.0600 & 0.0600 \\
\hline & KPI 7 & Kemasan Barang & 0.0196 & 0.0200 & 0.0200 & 0.0198 \\
\hline Pelayanan Purna Jual & KPI 8 & Frekuensi Kunjungan ke Pengguna & 0.0720 & 0.1000 & 0.0800 & 0.0800 \\
\hline Waktu Tanggapan & KPI 9 & Kecepatan Mengatasi Masalah & 0.0960 & 0.1000 & 0.0980 & 0.0990 \\
\hline \multirow[t]{3}{*}{\begin{tabular}{|l|} 
Laporan dan Administrasi \\
\end{tabular}} & KPI 10 & Frekuensi Pengiriman Laporan Reservasi & 0.0280 & 0.0350 & 0.0280 & 0.0280 \\
\hline & KPI 11 & Frekuensi Pengiriman Laporan SOH & 0.0280 & 0.0350 & 0.0280 & 0.0280 \\
\hline & KPI 12 & Konsisten Kerapihan Administrasi Kantor & 0.0300 & 0.0300 & 0.0300 & 0.0300 \\
\hline \multirow[t]{2}{*}{\begin{tabular}{|l|} 
Tingkat Pemenuhan \\
\end{tabular}} & KPI 13 & Mengikuti Aturan Pemerintah & 0.0500 & 0.0500 & 0.0500 & 0.0500 \\
\hline & KPI 14 & Pelanggaran Terhadap Aturan Pemerintah & 0.0500 & 0.0500 & 0.0500 & 0.0500 \\
\hline Sumber Daya & KPI 15 & $\begin{array}{l}\text { Tingkat Penggunaan Sumber Daya dari } \\
\text { Komunitas Lokal }\end{array}$ & NA & NA & NA & NA \\
\hline
\end{tabular}

\section{1) On-Time Services}

This criterion measures the timeliness of VHS supplier services to user-specified locations, which must be following the targets given.

\section{2) Safety}

This criterion measures supplier discipline to comply with all safety regulations determined by PT.X and measures how many safety violations were committed by suppliers.

\section{3) Quality}

This criterion measures the ability of suppliers to maintain the quality of goods provided by agreed standards and guarantee the authenticity of the goods.

\section{4) After Sales Services}

This criterion measures the ability of suppliers to provide technical ser vices in connection with the goods provided.

\section{5) Response Time}

This criterion measures how long the response time is given by

the supplier to each question and other communication related to shipping, prices, specifications, or requests for quotes for the items needed immediately.

\section{6) Reporting and Admin}

This criterion measures supplier discipline in providing routine reports related to the VHS process and administrative tidiness at the supplier's office.

\section{7) Compliance}

This criterion measures supplier discipline in complying with government regulations in full force.

\section{RESULT AND DISCUSSIONS}

\section{A. Determination of Criteria and Sub-Criteria}

The process of determining criteria and sub-criteria is based on the distribution of questionnaires to 3 respondents from the procurement department. The questionnaire included the selection of interests of each criterion and subcriterion that had been applied at PT X (Table 2).

In Table 2 it can be seen that there are 2 sub-criteria which according to respondents are not important, namely the frequency of training and workshop procurement and the speed of answering questions. Through interviews with respondents, the reason is explained because the training and workshop are only for certain types of items or if there is an 
The $6^{\text {th }}$ International Seminar on Science and Technology (ISST) 2020

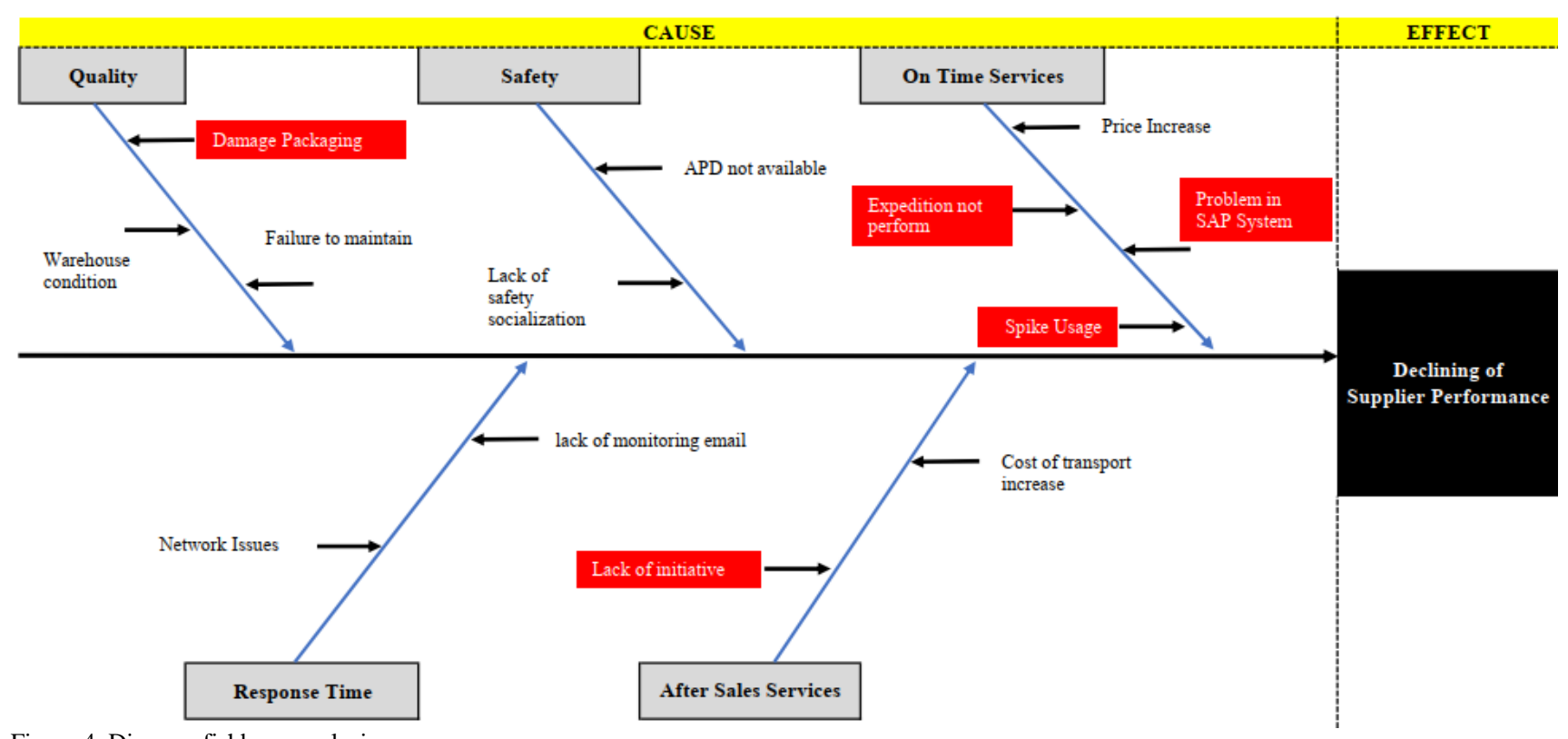

Figure 4. Diagram fishbone analysis.

Table 11

Scoring OMAX and traffic light system PT. AKM

\begin{tabular}{|c|c|c|c|c|c|c|c|c|c|c|c|c|c|c|c|c|}
\hline \multicolumn{2}{|c|}{ Performance Kriteria } & KPI 1 & KPI 2 & KPI 3 & KPI 4 & KPI 5 & KPI 6 & KPI 7 & PI & KPI 9 & 0 & & 12 & & 14 & KPI 15 \\
\hline \multicolumn{2}{|c|}{ Performance } & 0.1080 & 0.1080 & 0.0500 & 0.0500 & 0.1195 & 0.0600 & 0.0198 & 0.0800 & 0.0990 & 0.0280 & 0.0280 & 0.0300 & 0.0500 & 0.0500 & 0.0000 \\
\hline & 10 & 0.1500 & 0.1500 & 0.0500 & 0.0500 & 0.1200 & 0.0600 & 0.0200 & 0.1000 & 0.1000 & 0.0350 & 0.0350 & 0.0300 & 0.0500 & 0.0500 & 0.0000 \\
\hline & 9 & 0.1449 & 0.1449 & 0.0500 & 0.0500 & 0.1199 & 0.0600 & 0.0200 & 0.0971 & 0.0997 & 0.0340 & 0.0340 & 0.0300 & 0.0500 & 0.0500 & 0.0000 \\
\hline & 8 & 0.1397 & 0.1397 & 0.0500 & 0.0500 & 0.1197 & 0.0600 & 0.0200 & 0.0943 & 0.0994 & 0.0330 & 0.0330 & 0.0300 & 0.0500 & 0.0500 & 0.0000 \\
\hline $\mathrm{s}$ & 7 & 0.1346 & 0.1346 & 0.0500 & 0.0500 & 0.1196 & 0.0600 & 0.0200 & 0.0914 & 0.0991 & 0.0320 & 0.0320 & 0.0300 & 0.0500 & 0.0500 & 0.0000 \\
\hline $\mathrm{C}$ & 6 & 0.1294 & 0.1294 & 0.0500 & 0.0500 & 0.1194 & 0.0600 & 0.0200 & 0.0886 & 0.0989 & 0.0310 & 0.0310 & 0.0300 & 0.0500 & 0.0500 & 0.0000 \\
\hline $\mathrm{O}$ & 5 & 0.1243 & 0.1243 & 0.0500 & 0.0500 & 0.1193 & 0.0600 & 0.0200 & 0.0857 & 0.0986 & 0.0300 & 0.0300 & 0.0300 & 0.0500 & 0.0500 & 0.0000 \\
\hline $\mathrm{R}$ & 4 & 0.1191 & 0.1191 & 0.0500 & 0.0500 & 0.1191 & 0.0600 & 0.0200 & 0.0829 & 0.0983 & 0.0290 & 0.0290 & 0.0300 & 0.0500 & 0.0500 & 0.0000 \\
\hline \multirow[t]{4}{*}{ E } & 3 & 0.1140 & 0.1140 & 0.0500 & 0.0500 & 0.1190 & 0.0600 & 0.0200 & 0.0800 & 0.0980 & 0.0280 & 0.0280 & 0.0300 & 0.0500 & 0.0500 & 0.0000 \\
\hline & 2 & 0.1100 & 0.1100 & 0.0333 & 0.0333 & 0.1190 & 0.0600 & 0.0199 & 0.0773 & 0.0973 & 0.0280 & 0.0280 & 0.0300 & 0.0500 & 0.0500 & 0.0000 \\
\hline & 1 & 0.1060 & 0.1060 & 0.0167 & 0.0167 & 0.1190 & 0.0600 & 0.0197 & 0.0747 & 0.0967 & 0.0280 & 0.0280 & 0.0300 & 0.0500 & 0.0500 & 0.0000 \\
\hline & 0 & 0.1020 & 0.1020 & 0.0000 & 0.0000 & 0.1190 & 0.0600 & 0.0196 & 0.0720 & 0.0960 & 0.0280 & 0.0280 & 0.0300 & 0.0500 & 0.0500 & 0.0000 \\
\hline \multicolumn{2}{|l|}{ Score } & 1 & 1 & 10 & 10 & 6 & 10 & 1 & 3 & 6 & 3 & 3 & 10 & 10 & 10 & 0 \\
\hline \multicolumn{2}{|l|}{ Weight } & 0.106 & 0.031 & 0.024 & 0.177 & 0.023 & 0.171 & 0.012 & 0.089 & 0.102 & 0.037 & 0.013 & 0.007 & 0.124 & 0.004 & 0.080 \\
\hline \multicolumn{2}{|l|}{ Value } & 0.1063 & 0.0307 & 0.2368 & 1.7720 & 0.1384 & 1.7083 & 0.0121 & 0.2681 & 0.6108 & 0.1101 & 0.0403 & 0.0736 & 1.2373 & 0.0412 & 0.0000 \\
\hline \multicolumn{2}{|c|}{ Total Value } & \multicolumn{15}{|c|}{6.3862} \\
\hline
\end{tabular}

Table 12.

Recapitulation OMAX and traffic light system

\begin{tabular}{lllll}
\hline \hline Supplier & Total Value & Red & Yellow & Green \\
\hline PT. CUMS & 5.7483 & 8 & 0 & 6 \\
CV. SUN & 6.8414 & 2 & 6 & 6 \\
PT. AKM & 6.3862 & 6 & 2 & 6 \\
\hline \hline
\end{tabular}

updated change in the way of using the se items that require special training. Then from the respondents, there are additional criteria and sub-criteria that are considered important, namely resources and the level of use of resources from the local community.

\section{B. Determination of Interest Weight of Each Criterion and Sub-Criteria using AHP}

From the results of the distribution of questionnaire 1 (determining criteria and sub-criteria), the second questionnaire was distributed to 5 respondents from the procurement and maintenance department. Calculations are carried out using pairwise comparison matrices, so weights will be obtained for each criterion and sub-criteria. From Table 3 it can be seen that the results of weighting between criteria are obtained from questionnaire calculations that are mapped into the intensity of interests between criteria using a determinant matrix. The weight obtained is then calculated the ratio consistency value, where the hierarchy consistency ratio used is not more than $10 \%$ or 0.1 (Saaty, 1988). From the calculation results, the consistency ratio value is $1 \%$ $(<10 \%)$ which means that the data generated is valid or consistent so that no data collection is needed.

From Table 4 it can be seen that the results of weighting on each criterion in order from highest to lowest are in the quality criteria with a weight of 0.206 , safety with a weight of 0.201 , the accuracy of service with a weight of 0.137 , level of fulfillment with a weight of 0.128 , response time with a weight of 0.102 , after-sales service with a weight of 0.089 , 
The $6^{\text {th }}$ International Seminar on Science and Technology (ISST) 2020

July $25^{\text {th }} 2020$, Institut Teknologi Sepuluh Nopember, Surabaya, Indonesia

resources with a weight of 0.080 and report and administration with a weight of 0.058 .

The weighting analysis for the service accuracy criteria is that the delivery speed has a higher weight of 0.78 compared to the availability of goods with a weight of 0.22 . Weighting analysis for safety criteria, the highest weighting is owned by complying with safety regulations with a weight of 0.88 and while using PPE (Personal Protective Equipment) with a weight of 0.12 . Weighting analysis for quality criteria with a weight of 0.83 for the authenticity of the goods, a weight of 0.11 for quality complaints from users, and a weight of 0.06 for the packaging of the goods. Weighting analysis for a report and administrative criteria, the weighting of the frequency of reservation report submission is 0.64 , the weighting of the frequency of delivery of stock on hand reports is 0.23 , and consistent weight is neatness of office administration 0.13. Weighting analysis for compliance criteria, a weight of 0.97 for following government regulations, and a weight of 0.03 for violations of government rules. For after-sales service criteria, response time and resources do not have sub-criteria weights. After comparing with the prevailing assessment at PT X, the AHP weighting found that the ranking of each criterion was not following the provisions of the evaluation and assessment of supplier performance at PT. X. Quality is the first criterion that suppliers must meet.

\section{Calculation using OMAX and Traffic Light System Method}

OMAX calculation starts by making a standard assessment of the data obtained from the performance evaluation of the three VHS suppliers, namely PT. CUMS, CV. SUN and PT. AKM. Data is taken based on the results of the assessment period from January 2017 to December 2019.

From Table 5 it can be seen that the highest value of the performance of PT. CUMS in 2017 was 0.8552 and dropped to the lowest performance in 2018 which was 0.5935 . CV. SUN has the lowest performance in 2018 which is 0.8072 and the highest performance in 2019 is 0.9348 . While PT. AKM has the highest value in 2019 which is 0.8910 , but down in 2018 which is 0.7566 . More details can be seen in Figure 2.

The results of the performance analysis of the three suppliers will be used as a reference in calculations using the OMAX method.

\section{1) Calculation OMAX and Traffic Light System PT. CUMS}

In Table 6 and Table 7 can be seen KPI data obtained from PT. CUMS in the 2017-2019 period. For resource criteria, there is no value because it has never been included in KPI calculations by PT. X.

\section{2) Calculation OMAX and Traffic Light System CV. SUN}

From Table 8 you can see KPI data obtained from CV. SUN in the 2017-2019 period. For resource criteria, there is no value because it has never been included in KPI calculations by PT. X.

\section{3) Calculation OMAX and Traffic Light System PT. AKM}

From Table 10 can be seen KPI data obtained from PT. AKM in the 2017-2019 period. For resource criteria, there is no value because it has never been included in KPI calculations by PT.X.

Based on the results of data processing using OMAX seen in Tables 7, Table 9, and Table 11, then the performance of each of the suppliers can be described as a Table 12. In Table 12 it can be seen that the total value for the three suppliers reaches values between levels 4-7. This means that the achievement of performance has not been achieved even though the value is nearing the target.

\section{Analysis of Causes and Solutions using Fishbone Diagrams}

From Figure 3 it can be seen that the best performance is CV. SUN and the worst is PT. CUMS. Because it will then be analyzed the problem solving related to the decrease in performance of PT. CUMS by using a fishbone diagram.

Analysis of Figure 4 above shows that the most likely causes of declining supplier performance on service accuracy criteria are late expeditions in shipping, increased user requirements that exceed the estimated 3-month stock, and SAP system problems resulting in reservations received by suppliers after the delivery deadline has passed. While the quality criterion is the damage to the packaging when the item is received. In the after-sales service criteria, the cause is the lack of initiative to provide services with the principal.

\section{CONCLUSION}

The conclusions that can be drawn from this study are as follows: (1) From processing data using Analytical Hierarchy Process (AHP), the results obtained are the weighted value of each criterion which amounts to 8 with the lowest weight owned by the Report and Administration criteria 0.058, and the highest weight is owned by Quality 0.206. (2) From the combination of AHP and OMAX method calculations, weights are obtained for each criterion for the three suppliers where CV. SUN has the highest weighting of 6.8414 and PT. AKM 6.3862. PT. CUMS has the lowest weight of 5.7483. Then the OMAX calculation results in $\mathrm{CV}$ performance. SUN and PT. AKM is in the yellow area which means that the performance is nearing the target. While PT. CUMS is in the red area, which means that performance is really below the target set by the company and requires immediate improvement. (3) To find out the factors that cause a decline in the performance of PT. CUMS is then analyzed using a fishbone diagram. The analysis shows that 5 problems cause a decrease in performance, namely a late expedition to make deliveries, an increase in user needs, problems with the SAP system, damage to packaging when goods are received, and a lack of initiative to provide services with the principal. After knowing the problem and finding improvement recommendations, it is expected that the performance of PT. CUMS can meet company targets.

\section{REFERENCES}

[1] Aryandito Tjahjo Tamtomo, 2008. Pengukuran Produktivitas Proses Produksi PT. Halco dengan Menggunakan Alat Ukur OMAX (Objective Matrix). Jakarta: Universitas Indonesia. 
The $6^{\text {th }}$ International Seminar on Science and Technology (ISST) 2020

July $25^{\text {th }} 2020$, Institut Teknologi Sepuluh Nopember, Surabaya, Indonesia

[2] C. Elanchezhian, B. Vijaya Ramnath \& Dr.R. Kesavan. (2010). Vendor Evaluation Using Multi Criteria Decision Making Technique. International Journal of Computer Applications (0975 - 8887).

[3] Diah Nurul Hidayati, Irwan Sukendar, Nurwidiana. 2018. Implementation of supply chain management in supplier performance assessment using Analytical Hierarchy Process (AHP) Objective Matrix (OMAX) and Traffic Light System. Teknik Industri Unissula.

[4] Hesham Ahmed Hassan \& Mohammed Rady. (2015). A Model for Evaluating and Improving Supply Chain Performance.

[5] J.L. Riggs, production systems: planning, analysis, and control: New York: john willey and sons, 1986.

[6] Lisnuaril Anggarawati, Remba Yanuar Efranto, Dewi Hardiningtyas. 2018. Analisis Produktivitas pada Proses Produksi PG. Djombang
Baru dengan Metode Objective Matrix. Jurnal Rekayasa dan Manajemen Sistem Industri, Vol 6.

[7] Mayank Agrawal. (2017). 7 Special Purchasing Systems of Materials, Materials Management.

[8] Neela Ravindra Rajhans \& Rajesh Jagdish Dhake. (2013). Suppliers Delivery Performance Evaluation \& Improvement using AHP.

[9] Rahma Yanti. 2016. Analisa Pengukuran Kinerja Pemasok dengan Metode Analytical Hierarchy Process (AHP) dan Objective Matrix (OMAX) di PT. Panasonic Gobel Energy Indonesia (PT. PECGI).

[10] Uwe Clausen, Michael Ten Hompel \& Robert D Souza. (2019). Purchasing and Supply Management; Chapter 4; Holger Schiele; University of Twente Lecture Notes in Logistics.

[11] Zycus. A Quick and Effective Guide to Evaluating Supplier Performance. 